WERKZEUGE 


\section{PROF. \\ Über Beratungsresistenz}

von STEFAN RIEGER

«Ich will nicht beraten werden.

Beratung kommt für mich zu spät.»1

1 Heinz Strunk: Die Zunge Europas, Reinbek bei Hamburg 2016.

2 Sogar die Dissertation wird Gegenstand von Beratung. Vgl. dazu Hannes Beyerbach: Die juristische Doktorarbeit. Ein Ratgeber für das gesamte Promotionsuerfahren, 2. Aufl., München 2017.

3 Vgl. Friedrich Kittler: Das Subjekt als Beamter, in: Manfred Frank, Gérard Raulet, Willem van Reijen (Hg.): Die Frage nach dem Subjekt, Frankfurt/M. 1988, 401-420.
Vorbemerkung: Das folgende Professorenpsychogramm wird sich in absehbarer Zeit von selbst erledigt haben, betrifft es doch ein hochschulpolitisches Relikt. Es gilt einem beruflich habitualisierten Typus, der in der Grundausstattung männlich und in ausgewiesenen Sparten der Geisteswissenschaften situiert war. Vielleicht wäre daher das Präteritum die einzig taugliche Zeitform, um ihn zu beschreiben. Allen Veränderungen im universitären Personalgefüge zum Trotz weist dieser Typus gleichwohl ein gewisses Beharrungsvermögen auf. Seine hier kurz skizzierte Renitenz ist somit selbst renitent.

I.

Hochschulen verfügen inzwischen über ein breit gestreutes und hinreichend ausdifferenziertes Beratungsangebot. Dieses zielt auf sämtliche Statusgruppen und sorgt sich um unterschiedliche Anliegen. Vom wissenschaftlichen Schreiben über die psychosoziale Intervention in Krisenfällen bis zur Personalführung und der Verhandlung in Berufungsangelegenheiten oder der Optimierung der Lehre stehen eine Vielzahl von Coaching- und Beratungsangeboten zur Verfügung. ${ }^{2}$ Diese sind Ausdruck einer Veränderung der Hochschullandschaft, die unter Begriffen wie Ökonomisierung, Globalisierung, Internationalisierung und Professionalisierung einem Reformdruck von außen gerecht zu werden suchen. Es ist ein vor dem Hintergrund der Beratung einer ganzen Institution vielleicht vernachlässigbarer Aspekt, der im Folgenden in den Fokus gerückt werden soll: das professorale Subjekt, das Friedrich Kittler einmal unter dem Titel Das Subjekt als Beamter beschrieben hat. ${ }^{3}$ Von der eben nur vermeintlichen Wertschätzung dieses Berufstands distanziert sich ein Werbeslogan der in Bochum ansässigen Technischen Hochschule Georg Agricola 
auf eigentümliche Weise. Der Slogan erteilt der unhinterfragten Ordinarienseligkeit früherer Tage einen gehörigen Dämpfer, verweist er doch darauf, dass die Abkürzung Prof. an jener Institution nicht als Titular eines gesellschaftlich gut angesehenen Berufsstandes, sondern als Abkürzung für das Substantiv Profi steht. ${ }^{4}$

Es ist im Folgenden nicht um eine Charakterologie von Professoren als schrägen Käuzen oder genialisch zertreuten Sonderlingen zu tun, sondern darum, einen entsprechenden Habitus, sollte es ihn geben oder überhaupt je gegeben haben, als etwas aufzuzeigen, das aus systemischen Gründen mit der Beratung seine liebe Not hat. Um die damit verbundenen Überlegungen vor idiosynkratischen und anekdotischen Eigenbeobachtungen zu schützen, soll zunächst der Blick auf Universitäten als regelungsbedürftige Institutionen gerichtet werden. In einem Buch mit dem Titel Hochschule als Organisation wird die sehr grundsätzliche Frage danach gestellt, ob die Institution Universität den Organisationsprinzipien anderer Institutionen folgt oder ob sie eine Art Solitär mit eigenen Zugangsweisen und Gesetzmäßigkeiten darstellt. ${ }^{5}$ Deviantes Personal, so die These der Organisationssoziologie, befördert dabei das Argument der besonderen Institution.

Einerseits lassen sich Hochschulen also als spezifische Organisationen verstehen. Professoren stilisieren sich mitunter gerne als distinktive Gruppe von Devianten, welche nicht mit Managern oder sonstigen Berufsgruppen verglichen werden wollen. Akademische Forschung und Lehre werden als einzigartige Formen von Arbeitserledigung unter den höchst spezifischen Bedingungen der doxa und Organisationsweisen des wissenschaftlichen Feldes interpretiert. Darum entzieht sich die Hochschulorganisation einer vergleichenden Organisationsforschung. ${ }^{6}$

Die im Folgenden angerissene These zielt darauf ab, dass Universitäten und dort im Speziellen die traditionsreicheren unter den Fakultäten Biotope der Beratungsresistenz sind - und zwar nicht aus Gründen einer spezifischen Charakterologie der jeweiligen Personen, sondern aus Gründen der sehr eigenen Genese des Berufsstands. Beratungsresistenz ist damit zunächst zu beschreiben als ein Effekt der unterschwelligen Wirkmacht einer historischen Semantik, die etwas mit den Individualisierungsschüben des von Friedrich Kittler so genannten Aufschreibesystems I 800 zu tun hat. Forschung und wissenschaftliches Arbeiten werden im Zuge dieser Altlast einem Bereich angenähert, der in der Eigentümlichkeit eines starken, weil unverwechselbaren Individuums begründet liegt, einem Bollwerk des Stils und des eigenen Ausdrucks verpflichtet, den unsere Kultur habituell mit der Genialität des vornehmlich männlichen Künstlersubjekts assoziiert. Versuche, den verbeamteten Professor beraten zu wollen, müssen aus Gründen seines derart definierten Subjektstatus und Selbstentwurfes ins Leere laufen. Für die Literaturwissenschaft etwa ist auf ein bestimmtes Verständnis von Hermeneutik und damit seine Verankerung in bestimmten Sparten der Geistes- und Kulturwissenschaften als Katalysatoren dieses Prozesses zu verweisen. Es sind alte Disziplinen wie die
4 Vgl. auch die Homepage der Hochschule: www.coolstes-studiumder-welt.de, gesehen am 29.1.2018. 5 Zu diesem Alleinstellungsmerkmal der Universität vgl. Christine Musselin: Are Universities Specific Organisations?, in: Georg Krücken, Anna Kosmützky, Marc Torka (Hg.): Towards a Multiversity? Universities between Global Trends and National Traditions, Bielefeld 2007, 63-84.

6 Uwe Wilkesmann, Christian J. Schmid (Hg.): Hochschule als Organisation, Wiesbaden 2012, 8.

$7 \mathrm{Zu}$ dieser Formel vgl. Otto Friedrich Bollnow: Was heißt, einen Schriftsteller besser verstehen, als er sich selber verstanden hat?, in: Deutsche Vierteljahresschrift, Bd. 18 , Nr. 2, 1940, 117-138.

8 Vgl. dazu Johann Wilhelm Ritter: Physik als Kunst. Ein Versuch, die Tendenz der Physik aus ihrer Geschichte zu deuten. Zur Stiftungsfeyer der Königlichbaierischen Akademie der Wissenschaften am 28sten März 1806, München 1806; Hans-Jörg Rheinberger: Experimentelle Virtuosität, in: Caroline Welsh, Stefan Willer (Hg.): Interesse für bedingtes Wissen, Wechselbeziehungen zwischen den Wissenskulturen, München 2008, 331-342, sowie Hans-Jörg Rheinberger: Erkenntnisse in Wissenschaft und Kunst. Sind Künstler nicht auch Forscher und Forscher Künstler?, in: Peter Heintel, ders., Felix Tretter, Wolfgang Zinggl (Hg.): Wissenschaft: Kunst. Sind Künstler Forscher und Forscher Künstler?, Klagenfurt 2017, 111-128.

9 Vgl. Bernhard Fabian: Der Naturwissenschaftler als Originalgenie, in: Hugo Friedrich, Fritz Schalk (Hg.): Europäische Aufklärung. Herbert Dieckmann zum 6o. Geburtstag, München 1967, 47-68; Hendrik Anton Kramers: Physiker als Stilisten, in: Die Naturwissenschaften, Bd. 23, Nr. 19, 1935, 297-301.

10 Wolfgang Dreybrodt: (Ko)Autorenschaft, in: Forschung \& Lehre, Bd. 25, Nr. 1, 2018, online unter www.forschung-und-lehre.del wordpress/?page_id=298, gesehen am 29.1.2018. 
Werbung für die Technische Hochschule Georg Agricola in Bochum
Germanistik oder die Geschichtswissenschaft und eben nicht neuere wie die Gender Studies, die im Schlagschatten einer oft monomanischen Nähe zu ihrem Gegenstand stehen. Dieses hermeneutische Begehren findet seine Verdichtung im Anspruch Wilhelm Diltheys, den Dichter besser verstehen zu können als dieser sich selbst. ${ }^{7}$ Aber auch die Naturwissenschaften sind davon betroffen - dann etwa, wenn sie das Experiment als nicht angeleitete und nicht anleitbare Virtuosität veranschlagen - wie der romantische Naturwissenschaftler Johann Wilhelm Ritter das tut, indem er die Physik als Kunst behandelt, oder wie in dessen Nachfolge Hans-Jörg Rheinberger, der das Experiment in die Register der künstlerischen Praxis einträgt. ${ }^{8}$ Der Naturwissenschaftler wird (sich selbst) zum Originalgenie und die formelfreudige und abstrakte Physik zum Ort eines personal zurechenbaren Stilwillens. ${ }^{9}$

Angesichts der Praxis, Publikationen vor allem in den Naturwissenschaften durch immer größer werdende Schreibkollektive zu legitimieren, ist an prominenter Stelle, nämlich in der Rubrik <Standpunkt> der Zeitschrift Forschung \& Lehre des deutschen Hochschulverbands unter dem Titel (Ko)Autorschaft zu lesen: «Mit der Zahl der Autoren sinkt die Verantwortung des Einzelnen.»10 Sichtbar wird dabei aber noch etwas anderes, das die historische Semantik genialer Forscher trotz oder gerade wegen eines anderen Forschungs- und Publikationstyps im Spiel hält. Es ergibt sich eine Verteilung der Autorschaft, die unsere Kultur bisher nur schwer verkraftet. Und sie verkraftet sie umso schwerer, je mehr die Rede von der Exzellenz um sich greift. Aus Gründen der historischen Semantik und einer dieser geschuldeten Pragmatik, die im Schreiben <großer> Bücher ihren publizistischen Widerhall findet und die in Förderungsregularien wie dem Opus Magnum ein unzeitgemäßes Relikt darstellt, sind Exzellenz und Devianz noch immer und trotz der Prämierung von Kooperation unmittelbar aneinander gekoppelt. Der Wissenschaftsbetrieb sucht und findet diesen Typus. Für das Fach Medienwissenschaft war es Friedrich Kittler, der seine Devianz nicht nur im grandiosen Umgehen disziplinärer Kontexte und einer hochgradig ambitionierten Wissenschaftsprosa unter Beweis stellte, sondern der sie mit seinem exzessiven Zigarettenkonsum auch in Bezug auf universitäre

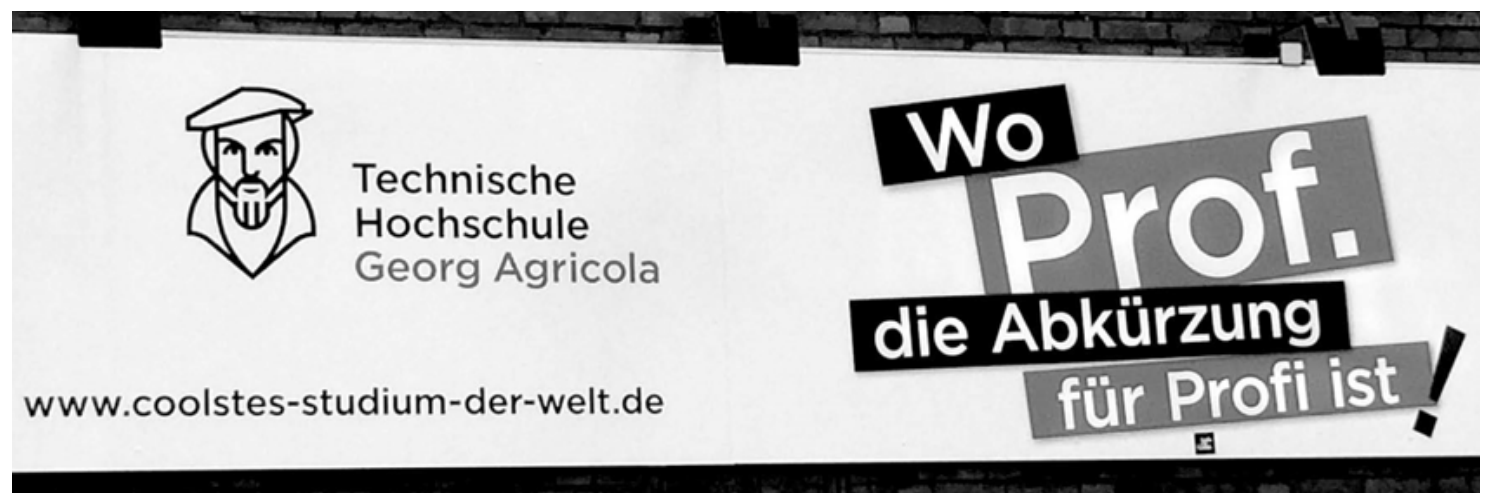


Hausordnungen auslebte. Im historiografischen Rückblick liefert das Habilitationsverfahren um das Buch Aufschreibesysteme 1800/1900 den Plot zu einer veritablen Widerstandsgeschichte. ${ }^{11}$

Diese Form des professoralen, verbeamteten und deshalb auch in Deutschland besonders wirkmächtigen Subjekts ist unzeitgemäß, aber immer noch in manchen Köpfen (wie eben das Beispiel der Förderungsform Opus Magnum zeigt). Devianten Menschen, die sich in der Organisation Universität alles selbst beibrachten oder für sich entschieden, eine Beibringung bestimmter Kompetenzen gar nicht erst nötig zu haben, diesen Subjekten Regeln von außen beibringen zu wollen, sie auf den Erwerb rationalisierter Arbeitstechniken und sachdienlicher skills zu verweisen, sie woraufhin auch immer beraten und coachen zu wollen, dieser Versuch misslingt. Wer diese Menschen beraten will, muss das Moment ihrer institutionell wie kulturgeschichtlich einprogrammierten Resistenz einrechnen. Ob bezogen auf die Lehre oder die Selbstverwaltung, auf die Personalführung oder die Bewirtschaftung der ihnen zugewiesenen Finanzmittel oder Büroräume: Bei Forschern und Forscherinnen, die sich selbst unter der Ägide des Genialitätsverdachts entworfen haben und auch haben entwerfen müssen, verpufft der an sie in Verwaltungsrundmails erteilte Hinweis, dass das Stoßlüften gegenüber dem Kipplüften eindeutig vorzuziehen sei. Derlei Ratschläge ziemen sich nicht für einen Berufsstand, der seine Professionalität gerade in Absetzung eines gewachsenen Berufsstands und seiner historischen Semantik erhält, wie der Slogan der Technischen Hochschule Georg Agricola insinuiert. Die Spezifik universitärer Rekrutierungspraktiken, die Logik der Berufung, die Strukturen der Unverbindlichkeit und der doch weitgehenden Sanktionsfreiheit professoralen Wirkens machen die Universität und ihre devianten Insassen zu etwas Spezifischem, selbst wenn sich die Figur des devianten Professors schon aus Generationengründen bald erledigt haben wird. Es scheint, als ob Berufung und Beratung sich ausschließen. ${ }^{12}$
11 Vgl. dazu Ute Holl, Claus Pias: Editorische Vorbemerkung zu: Aufschreibesysteme 1980/2010. In memoriam Friedrich Kittler, in: Zeitschrift für Medienwissenschaft, Nr. 6, 2012, $114 \mathrm{f}$.

12 Zu den Konsequenzen für die Universität der Zukunft aus Sicht eines posthumanistischen Wissenschaftsverständnisses vgl. Rosa Braidotti: Posthumanismus. Leben jenseits des Menschen, Frankfurt/M. 2014, insb. $176 \mathrm{ff}$. 